Control Iing the number of excited at ons fl owi ng into the reacti on chanber usi ng pul se- modul at ed i nduct i on ther mal pl asmas at at nospher i c pressure

\begin{tabular}{|l|l|}
\hline 著者 & Tanaka Yasunor i , Uesugi Yoshi hi ko, Sakut a T. \\
\hline $\begin{array}{l}\text { j our nal or } \\
\text { publ i cat i on ti tl e }\end{array}$ & Pl asma Sources Sci ence and Technol ogy \\
\hline vol une & 16 \\
\hline nunber & 2 \\
\hline page r ange & $281-289$ \\
\hline year & $2007-05-01$ \\
\hline URL & ht t p: //hdl . handl e. net /2297/6753 \\
\hline
\end{tabular}




\title{
Controlling the number of excited atoms flowing into the reaction chamber using pulse-modulated induction thermal plasmas at atmospheric pressure
}

\author{
Yasunori Tanaka $\nmid$, Y Uesugi, and T Sakuta \\ Division of Electrical Engineering and Computer Science, Kanazawa University, \\ Kakuma, Kanazawa 920-1192, JAPAN \\ E-mail: tanaka@ec.t.kanazawa-u.ac.jp
}

\begin{abstract}
.
Controlling the number of excited atoms into a reaction chamber was investigated in Ar pulse-modulated induction thermal plasma (PMITP) with molecular gases at atmospheric pressure. Such control is important in promoting the modification of substrate surfaces in thermal plasma processing. The coil current required for sustaining a PMITP was modulated by setting the firing angle of the metal-oxidesemiconductor field-effect transistor (MOSFET) in the inverter power supply used as a high-frequency power source. The radiation intensities of the excited atomic lines were measured using the optical emission spectroscopy (OES) technique to estimate the number of excited atoms flowing into the reaction chamber. In particular, we found an increase in the number of excited $\mathrm{Ar}$ and $\mathrm{H}$ atoms flowing into the reaction chamber in $\mathrm{Ar}$ or $\mathrm{Ar}-\mathrm{H}_{2}$ PMITPs as a result of the pulse modulation of the coil current.
\end{abstract}

Submitted to: Plasma Sources Sci. Technol.

$\nmid$ To whom correspondence should be addressed (tanaka@ec.t.kanazawa-u.ac.jp) 


\section{Introduction}

Inductively coupled thermal plasmas (ICTPs) approximately at atmospheric pressure have been widely used for plasma processing such as the syntheses of nanoparticles [1][3], diamond films [4, 5], fullerenes [6, 7], thermal barrier coatings [8, 9], and purification of Si [10]. The ICTPs provide an advantage of high enthalpy because of their high heavyparticle temperature and high pressure. Further, they are characterised by a high radical density and no contamination from the electrodes. However, it has been pointed out that thermal plasmas have excessively high enthalpy that is difficult to control during plasma processing. This uncontrollable high enthalpy causes some thermal damages on substrates and grown films. Therefore, a new technique is required for controlling the temperature of thermal plasmas for advanced thermal plasma processing.

To control this high enthalpy and temperature and to add a new function into the ICTP system, Ishigaki and our group have developed a pulse-modulated induction thermal plasma (PMITP) system [11]-[14]. This system can modulate the amplitude of the coil current that sustains induction thermal plasmas. The coil current modulation with a modulation frequency of approximately $100 \mathrm{~Hz}$ generates thermal plasmas under transient states. Further, it may enable control over the thermal plasma temperature and active particle density in the time domain. Furthermore, it can promote chemically and thermally non-equilibrium effects, even in high-power atmospheric pressure plasmas [15]-[17]. In particular, chemical non-equilibrium effects might provide new chemical reaction fields in high-power atmospheric pressure plasma, which can be useful in advanced material processing. The PMITP can also be used for the fundamental investigation of transient thermal plasmas, such as switching arcs in a circuit breaker $[18,19]$.

To date, we have investigated the dynamic behaviours of Ar PMITPs with the molecular gases $\mathrm{H}_{2}, \mathrm{~N}_{2}, \mathrm{O}_{2}$ and $\mathrm{CO}_{2}$. In these studies, we have focused on the high-temperature region in the plasma torch using both experimental and numerical approaches [15]-[20]. Both these approaches have shown that the minimum temperature of Ar thermal plasma with additional molecular gases can be controlled by setting the shimmer current level (SCL) while its maximum temperature in a modulation cycle is retained [15, 19, 20]. Moreover, it was found that the inclusion of $\mathrm{CO}_{2}$ causes a large temperature variation in the PMITP due to the modulation of the coil current [15, 19]. Further, based on this discussion, we found that the time-averaged temperature of the thermal plasma can be controlled using the pulse modulation of the coil current. On the other hand, recently, Ohashi et al experimentally applied atmospheric $\mathrm{Ar}-\mathrm{H}_{2}$ PMITP using a static induction transistor as the power source for $\mathrm{H}$ doping on $\mathrm{ZnO}$ $[21,22]$. They found that the irradiation of $\mathrm{Ar}-\mathrm{H}_{2}$ PMITP can successfully dope $\mathrm{H}$ atoms into $\mathrm{ZnO}$, thereby improving its photoluminescence. For this type PMITP material processing, knowledge of the dynamic behaviours downstream of the PMITP torch is required because material processing using PMITP is usually performed at this location.

This study addresses the experimental results from the dynamic behaviours of Ar 
PMITPs with molecular gases downstream of the plasma torch, i.e. at the inlet of the plasma reaction chamber. For comparison, we also studied the dynamic behaviours of the PMITP in the plasma torch region. An optical emission spectroscopic (OES) observation was carried out to elucidate the dynamic behaviour of excited atoms in the PMITP. From these measurements, we found that a greater number of excited Ar and $\mathrm{H}$ atoms flowing into the reaction chamber in $\mathrm{Ar}$ and $\mathrm{Ar}-\mathrm{H}_{2}$ PMITPs when compared with those in steady-state induction thermal plasmas.

\section{Parameters in pulse modulation of the coil current}

Figure 1 shows the schematic waveform of a typical pulse-amplitude-modulated coil current necessary for sustaining a PMITP. In this study, the PMITP is generated by using an amplitude-modulated coil current with a fundamental frequency of $450 \mathrm{kHz}$ and a modulation frequency of the order of ms. For the coil current modulation, we define the following four additional control parameters: 'higher current level (HCL)', 'lower current level (LCL)', 'on-time' (time period with the higher current level) and 'off-time' (time period with the lower current level). These parameters are also indicated in the figure. These parameters can be set independently from one another to obtain different dynamic states of a PMITP [20]. Further, we define the 'shimmer current level' (SCL) as the LCL/HCL. Controlling these parameters can provide the following expected features and effects to thermal plasmas: (1) control over time-averaged power, temperature and enthalpy of thermal plasmas in the time domain and (2) thermal and/or chemical nonequilibrium effects, even in high power-density atmospheric-pressure plasmas, can be introduced by the rapid application of an electric field under a transient state.

\section{Experimental setup and experimental conditions}

For sustaining a PMITP with high power-conversion efficiency, a metal-oxidesemiconductor field-effect transistor (MOSFET) inverter power supply was employed as the power source [12]. Figure 2 shows the electric circuit system for a PMITP used in the experiment. The system includes four sections: rectifier circuit, inverter circuit, impedance-matching circuit and induction plasma torch. The rectifier circuit has six diodes with a smoothing capacitor for generating DC power. This power is converted into AC power with a half-bridge inverter circuit including two MOSFET (rated $150 \mathrm{~V}$, 460 A) units. These units are operated at a fundamental frequency of $450 \mathrm{kHz}$. The MOSFET inverter section has considerably higher power-conversion efficiency - more than 85\% - with regard to the DC/AC inverter performance [23]. This efficiency is considerably greater than that of a conventional vacuum tube whose efficiency is below $60 \%$. The MOSFET inverter power supply was connected to an induction coil through a series of impedance-matching circuits. This system can modulate the amplitude of the $450-\mathrm{kHz}$ current with several hundred amperes by controlling the firing angle of the MOSFETs. 
Figure 3 shows the schematic diagram of the plasma torch and reaction chamber for PMITPs. The plasma torch comprises two coaxial quartz tubes with a length of 330 $\mathrm{mm}$. The inner tube has an inner diameter of $70 \mathrm{~mm}$. Between these two tubes, cooling water is allowed to flow in order to maintain the tube wall temperature at $300 \mathrm{~K}$. This plasma torch has an eight-turn induction coil around the quartz tube. Downstream of this plasma torch, a water-cooled reaction chamber is installed. This chamber is tapered to facilitate rapid cooling of the hot gas. Argon or an Ar-added gas mixture was supplied as a sheath gas along the interior of the inner quartz tube. The Ar gas flow rate was fixed at $100 \mathrm{slpm}(=\mathrm{l} / \mathrm{min})$. Pressure inside the chamber was fixed to $101325 \mathrm{~Pa}$ using an automatic feedback pressure controller. The 'on-time' was set to $10 \mathrm{~ms}$. The HCL was fixed at a value as that of the coil current during steady-state operation. Input power to the inverter power supply before modulation operation was fixed at $30 \mathrm{~kW}$.

Under these conditions, the SCL was modified by reducing the LCL with a fixed HCL in the modulation operation to determine the effect of pulse modulation on thermal plasmas. In addition, the 'off-time' was varied from $5 \mathrm{~ms}$ to $15 \mathrm{~ms}$ in order to investigate its effect on the dynamic behaviour of the PMITP. In this case, reducing the SCL and increasing the off-time decreased the output electric power from the inverter power supply. Four additional gases, namely, $\mathrm{H}_{2}, \mathrm{~N}_{2}, \mathrm{O}_{2}$ and $\mathrm{CO}_{2}$, were used to study the effects of the additional gases according to the gas type. These additional gases have been widely used in thermal plasma processing. The flow rate of these gases was 2.5 slpm.

\section{Spectroscopic observation system}

In this study, spectroscopic observations were carried out at two positions. One observation position is in the middle of the plasma torch, as denoted by $\mathrm{P}$ in figure 3 . This position corresponds to high temperature and high electric field strength regions in the plasma torch. The other position is $190 \mathrm{~mm}$ below the coil end in the reaction chamber; it is denoted by $\mathrm{R}$ in figure 3 . This position corresponds to the reaction chamber inlet, where the plasma material processing is usually performed.

Figure 4 shows the optical measurement systems. The right-hand side of this figure shows an optical measurement system performing time variation for three specific wavelengths. This system consists of optical fibre bundles, a camera, a monochromator, three photomultipliers and a digital oscilloscope. Light is guided through the camera and the optical fibre bundles towards the inlet of the monochromator. At the focal plane of the monochromator, three other optical fibre bundles are installed in order to capture light simultaneously at three different wavelengths.

At every other end of the three fibre bundles, photomultipliers are set in order to convert the radiation intensity of the captured light into electrical signals. These signals are recorded using a digital oscilloscope with a sampling time of $0.5 \mu \mathrm{s}$ in a time-dependent form. The wavelength width observed by this system is $1.0 \mathrm{~nm}$ for each detecting channel. The entire optical system was calibrated using a tungsten-halide 
lamp. In this experiment, we measured an Ar atomic spectral line at $751.465 \mathrm{~nm}$ and a neighbouring wavelength for continuum radiation at $755.0 \mathrm{~nm}$ to estimate the dynamic behaviour of the number of excited Ar atoms in the observation region.

Another spectroscopic observation system was also used for OES to measure the accumulated radiation intensity in the wide wavelength region. The left-hand side of this figure shows this system. It has a one-dimensional optical diode array that measures radiation intensities in the wavelength range of 610-780 $\mathrm{nm}$. The wavelength width observed by this system is $0.2 \mathrm{~nm}$ for each detecting diode. In this study, the exposure time is manually set as $3.0 \mathrm{~s}$ to obtain the time-accumulated radiation intensities of the spectral lines in the modulation cycle. These intensities are proportional to the time-averaged radiation intensities during the modulation cycle.

\section{Results and discussion}

\subsection{Coil current waveforms}

Figure 5 shows the measured waveforms of the modulated coil current peaks in Ar, Ar$\mathrm{H}_{2}$ and $\mathrm{Ar}-\mathrm{N}_{2}$ PMITPs as examples. The SCL is considered as a parameter with a fixed HCL. The amplitudes of the coil currents for these three types of gas inclusions can be modulated into almost square waveforms according to the pulsed control signal. This almost square waveform was also realised for the $\mathrm{Ar}-\mathrm{O}_{2}$ and $\mathrm{Ar}-\mathrm{CO}_{2}$ PMITPs. In this experiment, the step change in the coil current during the transition from LCL to HCL requires approximately $80 \mu \mathrm{s}$ for any gas plasma [20]. This time order for a step change in the coil current amplitude was confirmed to be equally smaller as the characteristic time required for a change in the heavy particle temperature in the thermal plasma. This characteristic time can be estimated to be 2-5 ms based on a numerical simulation of the PMITP assuming a local thermodynamic equilibrium condition [15] Thus, we can study the inherent dynamic behaviours of the thermal plasmas using PMITP.

\subsection{Dynamic behaviour of pulse-modulated induction thermal plasmas}

5.2.1. Relationship between radiation intensity and the number of excited atoms The radiation intensity $I_{\mathrm{Ar}}$ of an $\mathrm{Ar}$ atomic spectral line is proportional to the number of Ar atoms excited to an upper level $m$ in the observation region $\Omega$. This relationship is expressed as

$$
I=\int_{\Omega} N_{m} A_{m n} h \nu_{m n} d V=A_{m n} h \nu_{m n} \int_{\Omega} N_{m} d V=A_{m n} h \nu_{m n} \bar{N}_{m},
$$

where the subscripts $m$ and $n$ are the indices of the upper and lower levels, respectively, $N_{m}$ is the number of excited Ar atoms at an upper level $m, A_{m n}$ is the transition probability from level $m$ to level $n, h$ is the Planck's constant, and $\nu_{m n}$ is the frequency of emitted light, $d V$ is the differential volume and $\bar{N}_{m}$ is a whole number that denotes the number of the excited Ar atoms in the observation region $\Omega$. In this study, we measured the radiation intensity of an Ar atomic line at $751.465 \mathrm{~nm}$ whose upper level is 107054 
$\mathrm{cm}^{-1}$ and the configuration is $3 \mathrm{p}^{5}\left(2 \mathrm{P}_{1}^{0}{ }_{1 / 2}\right) 4 \mathrm{p}$. This upper level is not metastable, and $A_{m n}$ for a transition from the upper level to the lower level for this Ar spectral line is 4.02 $\times 10^{7} s^{-1}$ [24]. In this case, the variation in $I$ corresponds to that of $\bar{N}_{m}$. Therefore, the time variation in this radiation intensity results from a variation in the number of excited atoms in $\Omega$ in the PMITP.

5.2.2. Modulation in the number of excited atoms in the main thermal plasma region Figure 6(a) shows the pulsed control signal; figures 6(b)-6(d) show the time evolutions in the radiation intensities of the Ar spectral line at a wavelength of $751.465 \mathrm{~nm}$ measured in the main thermal plasma portion $\mathrm{P}$, i.e. in the middle of the coil region. The radiation intensities are normalised by those of $\mathrm{SCL}=100 \%$, i.e. values in the steadystate condition. This figure shows the periodic changes in the radiation intensities concomitant with the modulation of the coil current. This periodic change in the radiation intensities implies that $\bar{N}_{m}$ in $\Omega$ can be modulated through pulse modulation of the coil current. However, the waveform of radiation intensity is not a square waveform; approximately 2-5 $\mathrm{ms}$ are required for a rapid increase and a rapid decrease in the radiation intensity. This time order has already been roughly estimated by the numerical simulation of the PMITP assuming a local thermodynamic equilibrium [15]; however, the rapid increase in the radiation intensity obtained experimentally was less than that obtained by the numerical simulation. The difference between the experimental and numerically simulated results may be attributed to nonequilibrium effects. On the other hand, the additional gas only marginally affects the radiation intensity waveform in the main plasma region. This reason is inferred because the additional molecules almost completely dissociate into atoms, which might have similar thermodynamic and transport properties in this high-temperature region.

As shown in figure 6 , reducing the SCL decreases the minimum radiation intensity in a modulation cycle, for example, at approximately $t=10,25$ and $40 \mathrm{~ms}$ on the time axis. On the other hand, the maximum radiation intensity is recoverable at the final time during the 'on-time' of the on-operation at approximately $t=5,20,35$ and 50 $\mathrm{ms}$ on the time axis. Figure 7 shows the maximum and minimum radiation intensities of the Ar spectral line in a modulation cycle as a function of SCL. From this figure, it can be observed that the maximum of the radiation intensity marginally varies with the $S C L$, except for $S C L<50 \%$ in the $\mathrm{N}_{2}$ and $\mathrm{CO}_{2}$ inclusion cases. This marginal variation implies that a high-temperature plasma can almost be re-established during the on-operation. On the other hand, the minimum of the radiation intensity decreases with the SCL. From these results, only the minimum value of the number of excited particles can be varied during the modulation cycle by setting the $S C L$ as a fixed maximum value of the number of excited particles. 


\subsection{Reaction chamber region}

5.3.1. Increase in the excited atom flux by pulse-modulation Radicals and atoms flowing downstream of the plasma torch and then into the reaction chamber assume considerable importance for various material processes because these particles induce reactions during thermal plasma material processing. In this study, we investigate the excited atoms. Figure 8(a) shows the pulsed control signal, and figures 8(b)-8(e) show the time evolutions in the radiation intensities of the Ar line at $751 \mathrm{~nm}$ measured in the reaction chamber $\mathrm{R}$ for a pure Ar PMITP versus time $t$. The condition SCL $=100 \%$ corresponds to the unmodulated operation condition. In this case, only a few fluctuations can be observed in the radiation intensity. On the other hand, for the modulation condition of $S C L<100 \%$, some periodical peaks are observed in the radiation intensity waveform according to the pulsed control signal. For example, for $S C L=85 \%$, peaks in the radiation intensity waveform can be observed at around $t=$ 15, 30 and $45 \mathrm{~ms}$. These periodical peaks increase when the SCL decreases from $85 \%$ to $69 \%$. In the case of $S C L \leq 69 \%$, periodical peaks are clearly visible at approximately $t=$ 5, 20, 35 and $50 \mathrm{~ms}$. These waveforms are significantly different from those measured in the plasma torch provided in the previous section. In particular, the results show that the peak value of the radiation intensity at SCL $<100 \%$ is considerably greater than that at SCL $=100 \%$. In other words, the instantaneous peak magnitude of the number of excited Ar atoms flowing into the reaction chamber increases due to the pulse modulation of the coil current.

A similar increase in the peak of the radiation intensity is observed for $\mathrm{Ar}-\mathrm{H}_{2}$ PMITP. Figure 9(a) shows the pulsed control signal, and figures 9(b)-9(e) show the radiation intensities of the $\mathrm{Ar}$ line measured at position $\mathrm{R}$ in $\mathrm{Ar}-\mathrm{H}_{2}$ PMITP. In this case, the waveform of the radiation intensity exhibits steep periodical peaks at SCL $=84 \%$ and $66 \%$. Furthermore, the peak of the radiation intensity increases due to the modulation of the coil current for $S C L=84 \%$ and $66 \%$ as compared to $S C L=$ $100 \%$. Further, the peak value of the radiation intensity decreases when the SCL is reduced from $66 \%$ to $54 \%$. On the other hand, for Ar- $\mathrm{N}_{2}$ PMITP, figure 10 shows the time evolution in the radiation intensity of the Ar line measured at position $\mathrm{R}$. The characteristic peak in the radiation intensity is only marginally apparent in Ar- $\mathrm{N}_{2}$ PMITP for any SCL value. This may be approximately inferred as follows: Inclusion of $\mathrm{N}_{2}$ may reduce the plasma region mainly due to the electron energy consumption for the dissociation of $N_{2}$. As a result, the periodic radiation intensity variation in the Ar spectral line could not be observed from the window provided in the experimental setup. A high-speed video captured during another experiment also confirmed that the tail portion of the Ar- $\mathrm{N}_{2}$ PMITP did not reach downstream of the reaction chamber. The smaller plasma region in the $\mathrm{N}_{2}$ inclusion case as compared to pure Ar plasma can generally be deduced from the numerical simulation of thermal plasmas [18]. This simulation reveals the high specific heat and high thermal conductivity of the Ar- $N_{2}$ thermal plasmas because

of the $\mathrm{N}_{2}$ dissociation reaction at temperatures in the range of $6000-7500 \mathrm{~K}$; this results 
in the plasma region to shrink when compared with pure Ar thermal plasma [18].

5.3.2. Effects of shimmer current level and additional gas We investigated the effects of SCL on the increased number of excited atoms flowing into the reaction chamber. Figure 11(a) shows the peak values of the radiation intensities as a function of SCL for different gas PMITPs with an off-time of $5 \mathrm{~ms}$. Each radiation intensity is normalised by that at SCL $=100 \%$ for the gases of each type. This figure shows that a decrease in the SCL from 100\% increases the radiation intensity peak value for Ar PMITP. Such an increase in the radiation intensity peak value cannot be obtained in those measured in the main thermal plasma region, as shown in figures 6 and 7 . This increase can be inferred from the following facts: A rapid increase in the coil current amplitude can induce a rapid input energy to the plasma; then, it may rapidly cause dissociations of the molecular particles and ionizations of particles in the plasma. These rapid dissociations and ionizations appear to increase the pressure of the plasma; it can produce a rapid increase in momentum of the gas flow from the plasma torch to the reaction chamber. This inference needs to be confirmed by a numerical simulation of the PMITP in the near future.

On the other hand, for Ar- $\mathrm{H}_{2}$ PMITP, decreasing the SCL to $70 \%$ also increases the radiation intensity of the Ar spectral line. However, a further decrease in the SCL reduces the peak value of the radiation intensity, as shown in figure 11(a). For Ar- $\mathrm{O}_{2}$ and $\mathrm{Ar}-\mathrm{CO}_{2}$ PMITPs, a tendency similar to that of $\mathrm{Ar}-\mathrm{H}_{2}$ can be observed. In the $\mathrm{N}_{2}$ inclusion case, decreasing the $S C L$ does not increase the radiation intensity. The reason of this difference is difficult to explain. A numerical simulation needs to be conducted that includes the reaction chamber region and the effect of reaction rate [25].

Such an increase in the radiation intensity corresponds to an increase in the number of excited atoms flowing into the reaction chamber. Therefore, the abovementioned results indicate that the pulse modulation of the coil current can increase the instantaneous number of excited Ar atoms into the reaction chamber.

5.3.3. Off-time effect The off-time as well as SCL is also one of parameters of the PMITP. To study the effect of off-time on the abovementioned increase in the radiation intensity, it was increased from $5 \mathrm{~ms}$ to $15 \mathrm{~ms}$ with a fixed on-time of $10 \mathrm{~ms}$. Figures 11(b) and 11(c) show the peaks in the radiation intensities at off-times of $10 \mathrm{~ms}$ and 15 ms, respectively. In the case of Ar PMITP at off-times of 5, 10 and $15 \mathrm{~ms}$, we find that the radiation peak increases when the SCL reduces from $100 \%$ to approximately $70 \%$. Such an increase in the radiation intensity peak is apparent in $\mathrm{Ar}$ and $\mathrm{Ar}-\mathrm{H}_{2}$ PMITP for off-times of $5-15 \mathrm{~ms}$ for $70 \%<\mathrm{SCL}<100 \%$. However, for additional gas inclusion cases of $\mathrm{O}_{2}, \mathrm{~N}_{2}$ and $\mathrm{CO}_{2}$, the radiation intensity does not always increase even for $70 \%<\mathrm{SCL}$ $<100 \%$, particularly for a greater value of off-time. Such dependence of the normalized radiation intensity peak on the off-time for $S C L=80 \%$ is evident in figure 12. From this figure, we can notice that the normalized radiation intensity peak is visibly greater than unity for any value of off-time for Ar and Ar- $H_{2}$ PMITPs. On the other hand, for 
Ar- $\mathrm{O}_{2}, \mathrm{Ar}-\mathrm{N}_{2}$ and $\mathrm{Ar}-\mathrm{CO}_{2}$, the normalized radiation intensity peak for a greater value of off-time is generally smaller or equal to unity. This might be because the inclusions of these molecular gases shrink the thermal plasma, as previously mentioned. This can be attributed to the large electron energy losses arising mainly from dissociation reactions of molecules.

\subsection{Increases of time-averaged numbers of $A r$ and $H$ excited atoms}

The previous section explained that the instantaneous peak value of the numbers of the Ar excited atoms flowing into the reaction chamber can be increased by pulse modulation of the coil current for Ar and Ar- $\mathrm{H}_{2}$ PMITPs. However, the time-averaged value of the number of excited atoms, rather than its instantaneous value, might also be important for some material processes because material processes require a greater amount of time for the pulse-modulation cycle. Therefore, we measured the accumulated radiation intensities for some lines at wavelengths of 610-780 nm with a large exposure time of $3.0 \mathrm{~s}$ in order to estimate the time-averaged number of the excited atoms. For this measurement, we used a one-dimensional diode array system shown on the left-hand side of figure 4. This exposure time duration has 200 cycles of coil current modulation.

Figure 13 shows the accumulated radiation intensities from $\mathrm{Ar}-\mathrm{H}_{2}$ PMITP at onand off-times of $10 \mathrm{~ms}$ and $5 \mathrm{~ms}$, respectively. Several Ar atomic lines are visible in the wavelength range of $610-780 \mathrm{~nm}$. The $\mathrm{H}-\alpha$ atomic line can also be found at 656.285 $\mathrm{nm}$; its intensity is in proportion to the number of excited $\mathrm{H}$ atoms at $97492 \mathrm{~cm}^{-1}$. The number of $\mathrm{H}$ atoms is a very important factor for several material processing [22]. As shown in the figure, these accumulated radiation intensities vary markedly with the SCL.

Figure 14 shows the accumulated radiation intensities of the Ar line at $751.465 \mathrm{~nm}$ and the $\mathrm{H}-\alpha$ line at $656.285 \mathrm{~nm}$ versus SCL in $\mathrm{Ar}$ and $\mathrm{Ar}-\mathrm{H}_{2}$ PMITPs. Decreasing the SCL increases the accumulated radiation intensity of the Ar spectral line for $\mathrm{Ar}$ PMITP. For Ar- $\mathrm{H}_{2}$ PMITP, the accumulated radiation intensities of the Ar and $\mathrm{H}-\alpha$ spectral lines increase when the SCL reduces from 100\% to 60\%, although they decrease when the SCL is reduced from $60 \%$ to $40 \%$. The abovementioned results indicate that the pulse modulation of the coil current can increase the time-averaged number of the excited particles that flow into the reaction chamber.

\section{Conclusions}

Spectroscopic observations were performed downstream of the plasma torch of a pulsemodulated induction thermal plasma (PMITP) system to investigate the effect of pulse modulation of coil current on the excited particle flux into the reaction chamber. For this, the time variation in the radiation intensity of an Ar line at $751.465 \mathrm{~nm}$ was measured. Four test gases $-\mathrm{H}_{2}, \mathrm{O}_{2}, \mathrm{~N}_{2}$ and $\mathrm{CO}_{2}$ - were selected as the additional gases. The results revealed that the instantaneous peak value and time-averaged value of the 
radiation intensities of the spectral lines emitted from the excited particles increased due to the pulse modulation of the coil current for $\mathrm{Ar}$ and $\mathrm{Ar}-\mathrm{H}_{2}$ PMITPs. Consequently, the numbers of excited $\mathrm{Ar}$ and $\mathrm{H}$ atoms flowing into the reaction chamber increased due to pulse modulation. This increase might improve the interest in PMITP applications.

\section{Acknowledgments}

The authors would like to express their sincere gratitude to Professor Tadahiro Sakuta, Department of Electrical and Electronic Engineering, Kanazawa University, for his encouragement and instructive suggestions regarding this study during his life. The authors would also like to thank Mr M Katayama and Mr S Hida for their assistance in performing the experiments.

\section{References}

[1] Girshick S L, Chiu C P, Muno R, Wu C Y, Yang L, Singh S K and McMurry P H 1993 Thermal plasma synthesis of ultrafine iron particles J. Aerosol Sci. 24 367-82

[2] Ishigaki T, Oh S M, Li J G and Park D W 2005 Controlling the synthesis of TaC nanopowders by injecting liquid precursor into RF induction plasma Sci. \& Technol. Advanced Mater. 6 111-8

[3] Shigeta M, Watanabe T and Nishiyama H 2004 Numerical investigation for nano-particle synthesis in an RF inductively coupled plasma Thin Solid Films 457 192-200

[4] Berghaus J O, Meunier J L and Gitzhofer F 2004 Monitoring and control of RF thermal plasma diamond deposition via substrate biasing Meas. Sci. Technol. 15 161-4

[5] Matsumoto S, Hino M and Kobayashi T 1987 Synthesis of diamond films in a rf induction thermal plasma Appl. Phys. Lett. 51 737-9

[6] Wang C, Inazaki A, Shirai T, Tanaka Y, Sakuta T, Takikawa H and Matsuo H 2003 Effect of ambient gas and pressure on fullerene synthesis in induction thermal plasma Thin Solid Films $42541-8$

[7] Todorovic-Markovic B, Markovic Z, Mohai I, Karoly Z, Gal L, Foglein K, Szabo P T and Szepvolgyi J 2003 Efficient synthesis of fullerenes in RF thermal plasma reactor Chem. Phys. Lett. 378 434-9

[8] Huang H, Eguchi K and Yoshida T 2003 Novel structured yttria-stabilized zirconia coatings fabricated by hybrid thermal plasma spraying Sci. \& Technol. Advanced Mater. 4 617-22

[9] Chen W R, Wu X, Marple B R and Patnaik P C 2005 Oxidation and crack nucleation/growth in an air-plasma-sprayed thermal barrier coating with NiCrAlY bond coat Surface 83 Coatings Technol. 197 109-15

[10] Benmansour M, Nikravech M, Morvan D, Amouroux J and Chapelle J 2004 Diagnostic by emission spectroscopy of an argon-hydrogen RF inductive thermal plasma for purification of metallurgical grade silicon J. Phys. D: Appl. Phys. 37 2966-74

[11] Ishigaki T, Xiaobao F, Sakuta T, Banjo T, Shibuya Y 1997 Generation of pulse-modulated induction thermal plasma at atmospheric pressure Appl. Phys. Lett. 71 3787-9

[12] Sakuta T, Tanaka Y, Hashimoto Y, Katsuki M 2002 Novel system of an inductively coupled thermal plasma with pulse amplitude modulation of electromagnetic field Electr. Eng. Japan 138 26-33

[13] Sakuta T, Tanaka Y, Paul K C, Hossain M M, Ishigaki T 2000 Non-equilibrium effects in pulse modulated induction thermal plasma for advanced processing Trans. Mater. Res. Soc. Japan $\mathbf{2 5}$ $35-8$

[14] Tanaka Y and Sakuta T 2000 Measurement of dynamic response time in pulse modulated thermal plasma Trans. Mater. Res. Soc. Japan 25 293-6 
[15] Hossain M M, Tanaka Y and Sakuta T 2003 Transient nature of argon and molecular gasseeded argon inductive thermal plasmas in pulse amplitude modulation approach Trans. IEE of Japan123-PE 1333-49

[16] Hossain M M, Tanaka Y and Sakuta T 2003 Dynamic responses of $\mathrm{Ar}_{-} \mathrm{CO}_{2}$ and Ar- $\mathrm{N}_{2}$ induction thermal plasmas in pulse modulation approach: a numerical analysis Thin Solid Films 435 $19-26$

[17] Tanaka and Sakuta T 2004 Time-dependent two-dimensional chemical non-equilibrium modeling of $\mathrm{Ar}-\mathrm{N}_{2}$ pulse-modulated induction thermal plasma at atmospheric pressure for material processing Trans. Mater. Res. Soc. Japan 29 3403-6

[18] Tanaka Y and Sakuta T 2002 Investigation of plasma-quenching efficiency of various gases using induction thermal plasma technique: effect of various gas injection on Ar thermal ICP J. Phys. D: Appl. Phys. 35 2149-58

[19] Tanaka Y and Sakuta T 2003 Stable operation region and dynamic behavior of pulse modulated Ar thermal plasma with different molecular gases Electr. Eng. Japan 143 1-11

[20] Tanaka Y and Sakuta T 2003 Temperature control of Ar induction thermal plasma with diatomic molecular gases by pulse-amplitude modulation of coil-current Plasma Sources Sci. E Technol. $1269-77$

[21] Ohashi N, Ishigaki T, Okada N, Sekiguchi T, Sakaguchi I and Haneda H 2002 Effect of hydrogen doping on ultraviolet emission spectra of various types of $\mathrm{ZnO}$ Appl. Phys. Lett. 80 2869-71

[22] Ohashi N, Ishigaki T, Okada N, Taguchi H, Sakaguchi I, Hishita S, Sekiguchi T and Haneda H 2003 Passivation of active recombination centers in $\mathrm{ZnO}$ by hydrogen doping J. Appl. Phys. 93 6386-92

[23] Sakakibara Y, Katagiri G, Toraguchi M and Sakuta T 1998 Generation of large area inductively coupled plasma at atmospheric pressure with high efficiency Proc. 4th Asia-Pacific Conf. on Plasma Sci. $\&$ Technol. p 103

[24] NIST Atomic Spectra Database, National Institude of Standards and Technology, http://www.nist.gov/

[25] Tanaka Y 2005 Time-dependent two-dimensional two-temperature chemical non-equilibrium model of $\mathrm{Ar}-\mathrm{N}_{2}$ pulse modulated induction thermal plasmas: Influence of coil-current modulation on thermal non-equilibrium Proc. 18th Int. Symp. Plasma Chem. ISPC-18 No.ISPC-85 on CD-ROM 


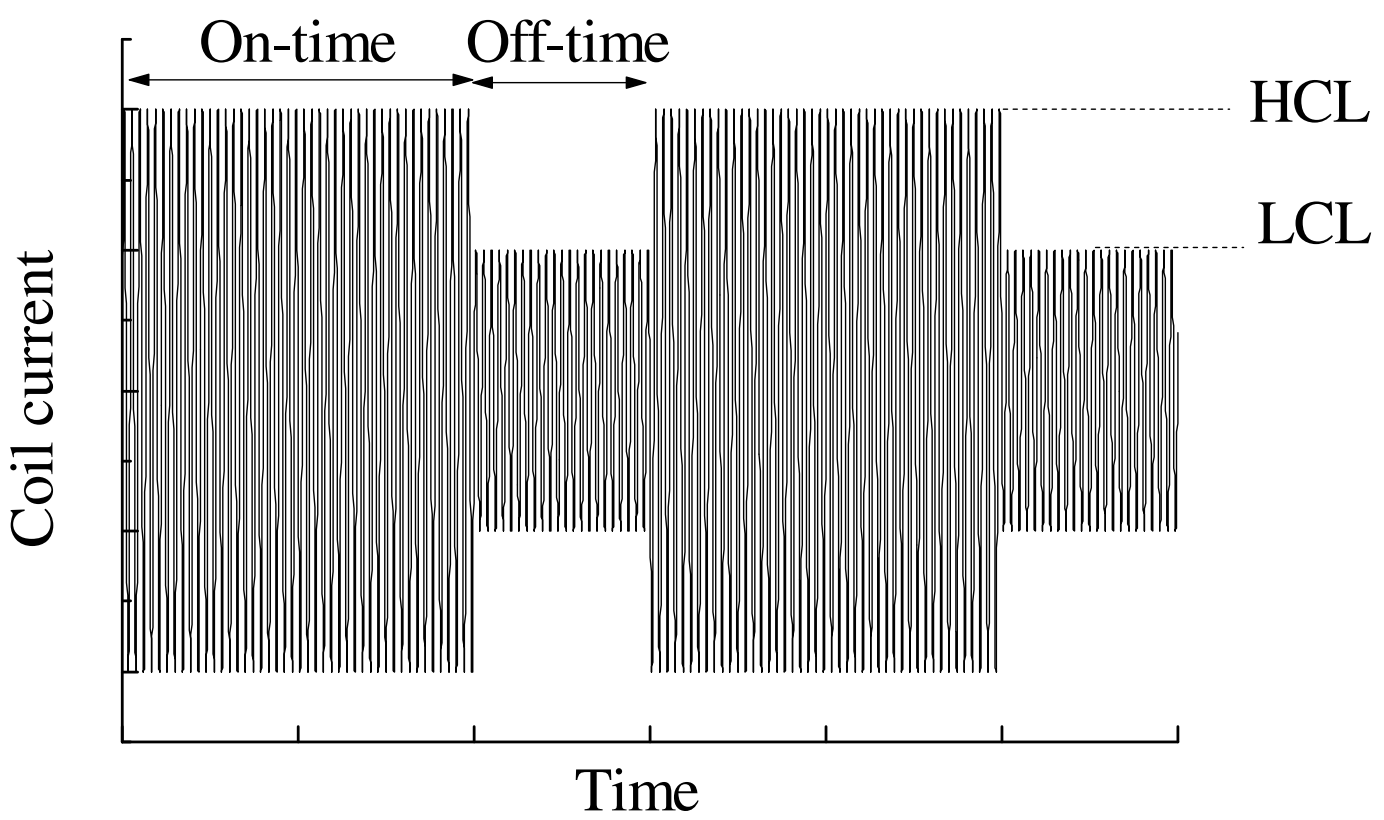

Figure 1. Pulse-modulated coil current and definition of control parameters.

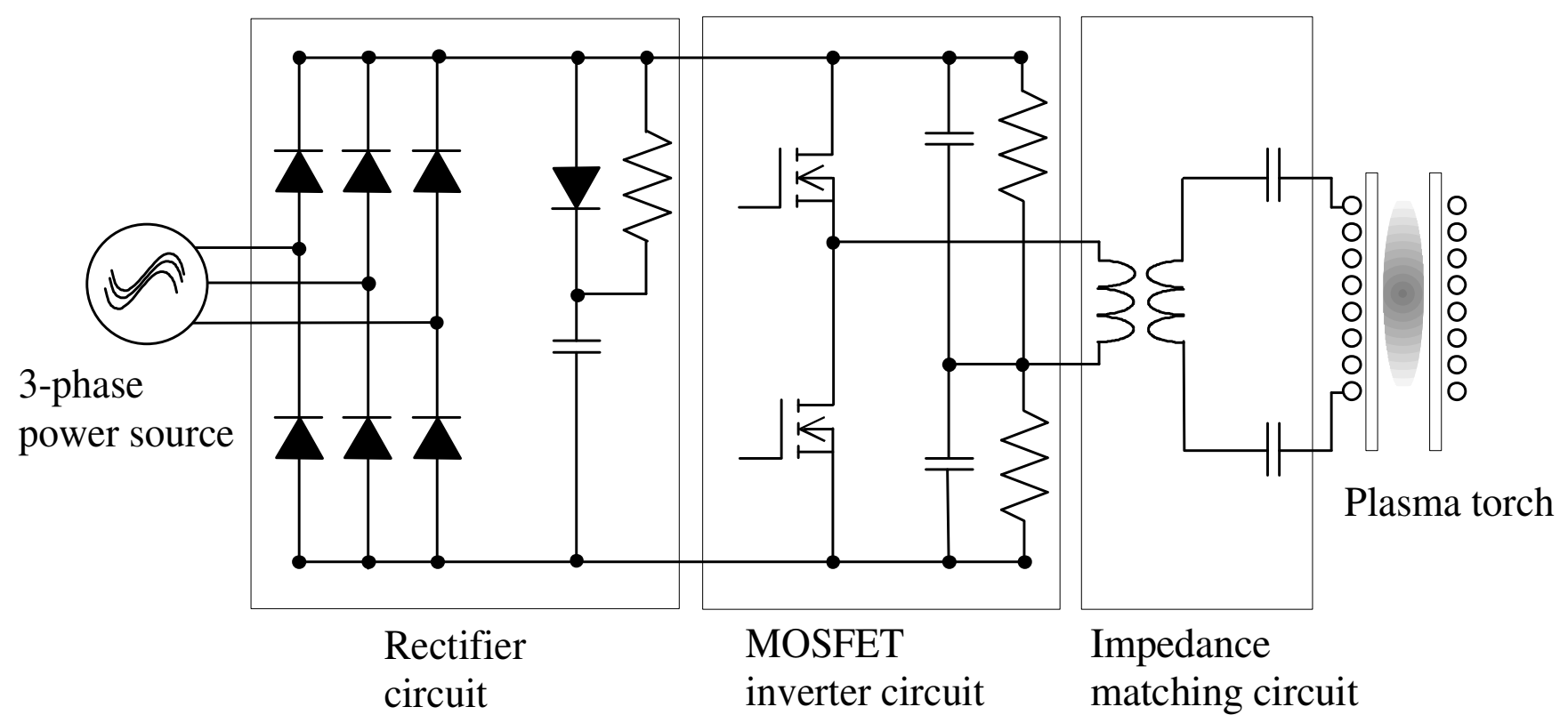

Figure 2. Electric circuit for pulse-modulated induction thermal plasmas. 


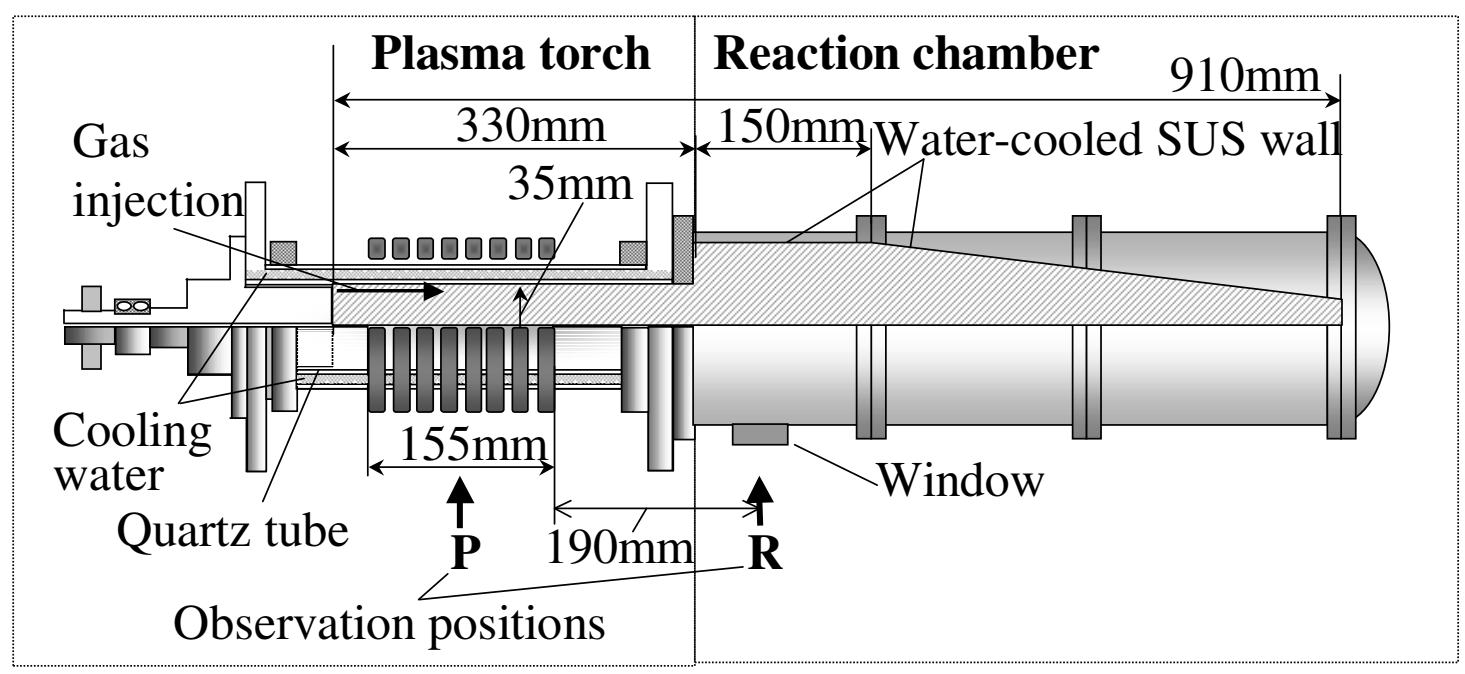

Figure 3. Configuration of the plasma torch and reaction chamber with spectroscopic observation positions.

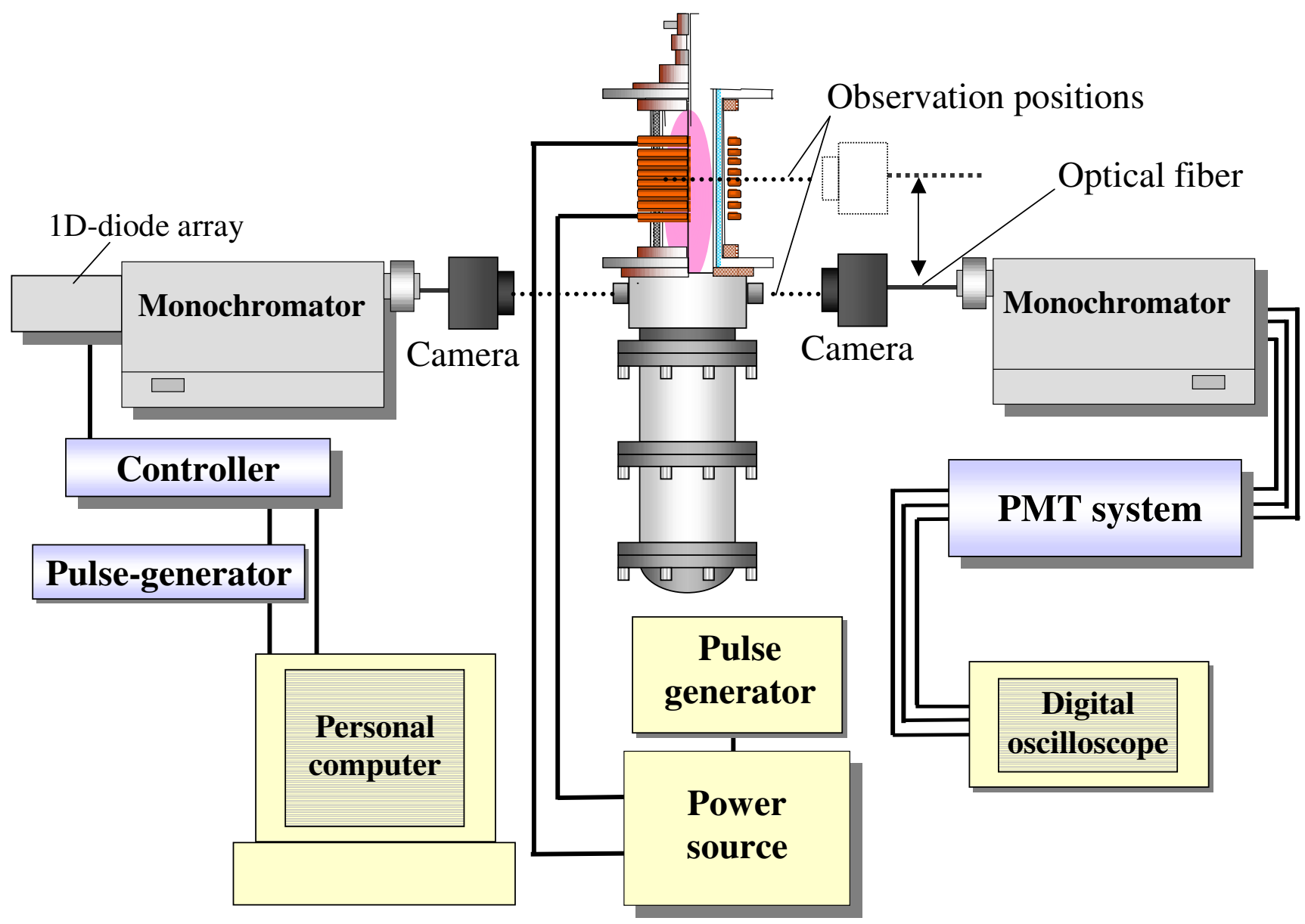

Figure 4. Time-dependent optical emission spectroscopy system for three wavelengths and a one-dimensional diode array system for spectroscopic observation. 

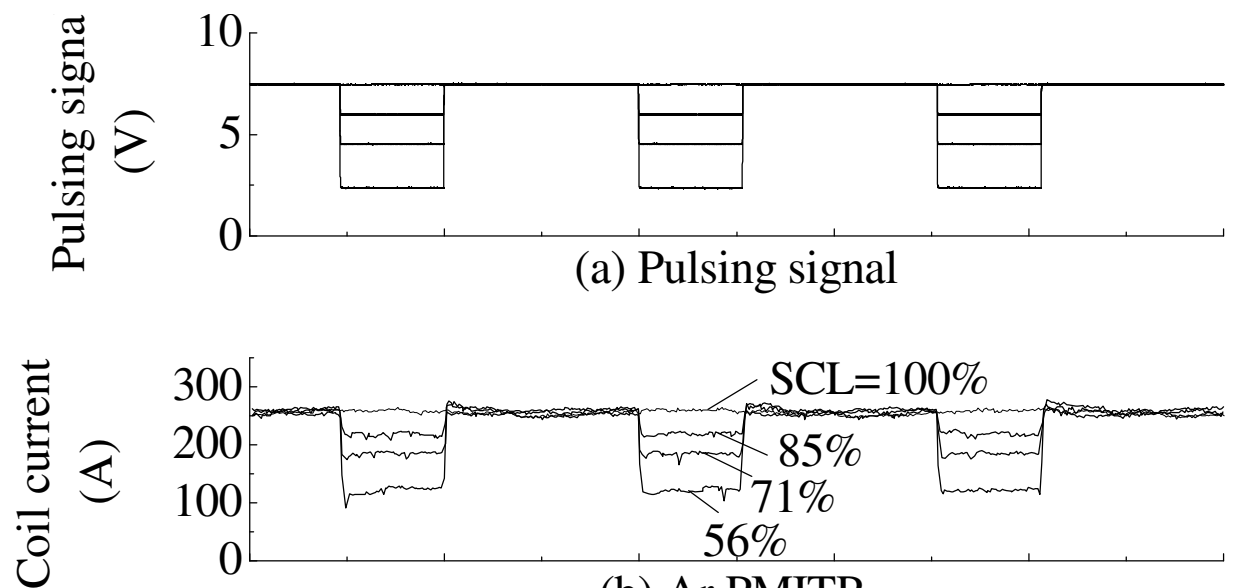

(b) Ar PMITP.

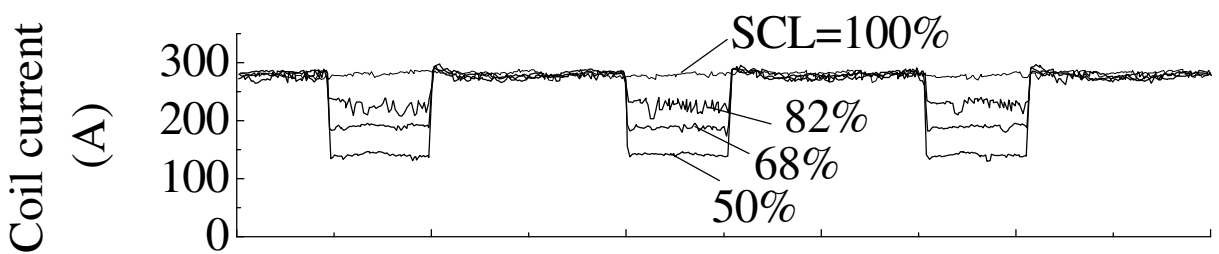

(c) Ar- $\mathrm{H}_{2}$ PMITP.

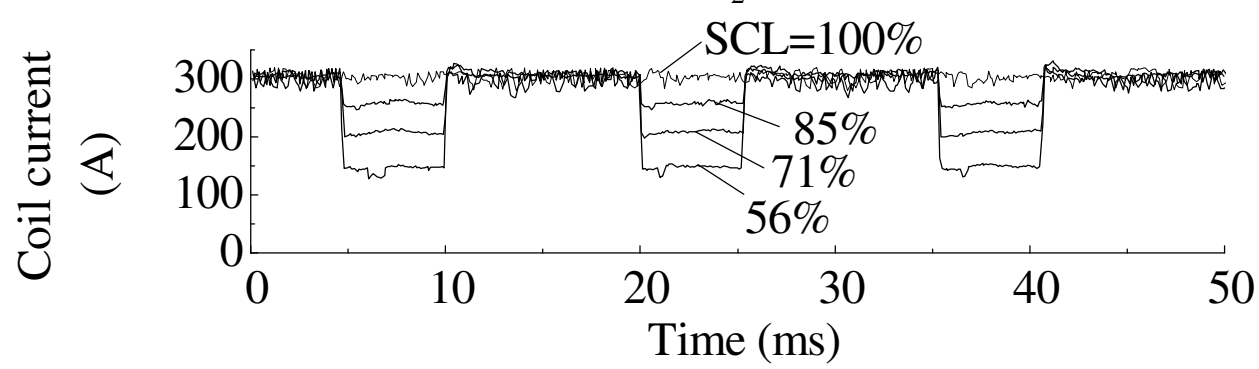

(d) Ar- $\mathrm{N}_{2}$ PMITP.

Figure 5. Measured coil current waveforms. (a) Pulsed signal. Waveforms for (b) Ar PMITP, (c) Ar- $\mathrm{H}_{2}$ PMITP and (d) Ar- $\mathrm{N}_{2}$ PMITP. Pressure is $101325 \mathrm{~Pa}$. The Ar and additional gas flow rates are $100 \mathrm{slpm}$ and 2.5 slpm, respectively. A higher current level (HCL) is fixed at that at $30 \mathrm{~kW}$ for unmodulated operation. The shimmer current level (SCL) is considered as a parameter. 

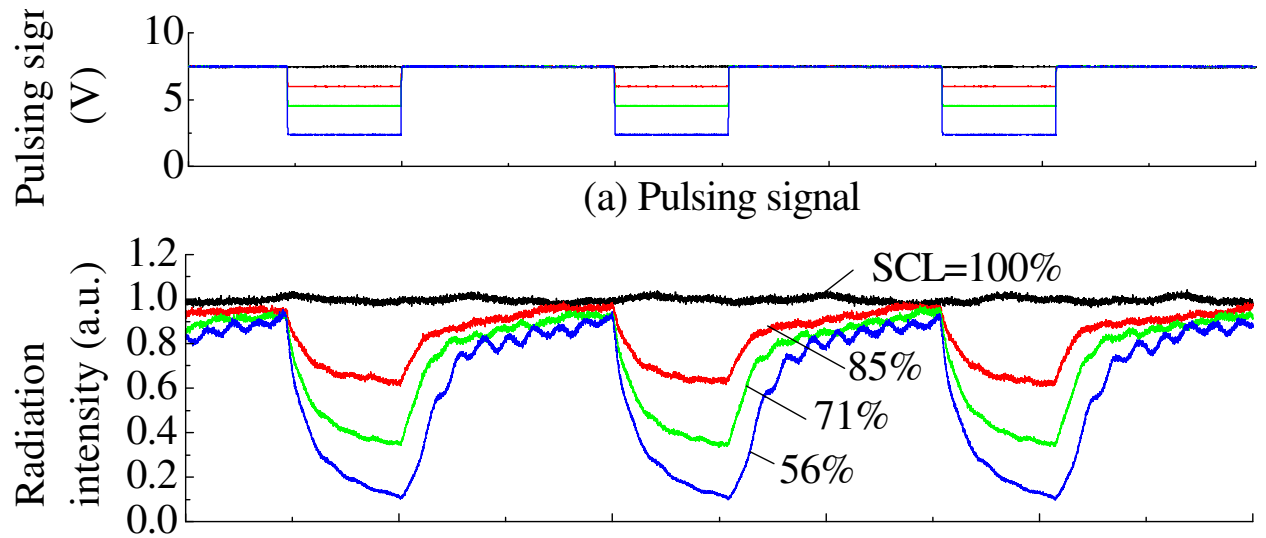

(b) Ar PMITP.

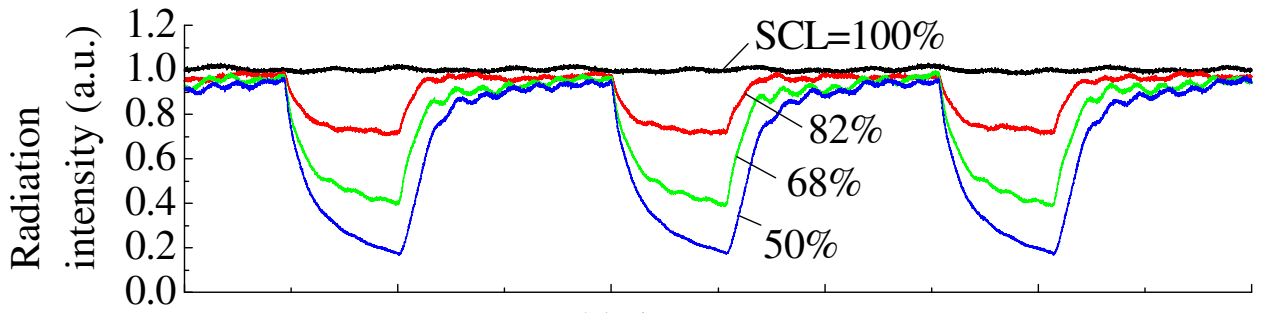

(c) $\mathrm{Ar}-\mathrm{H}_{2}$ PMITP.

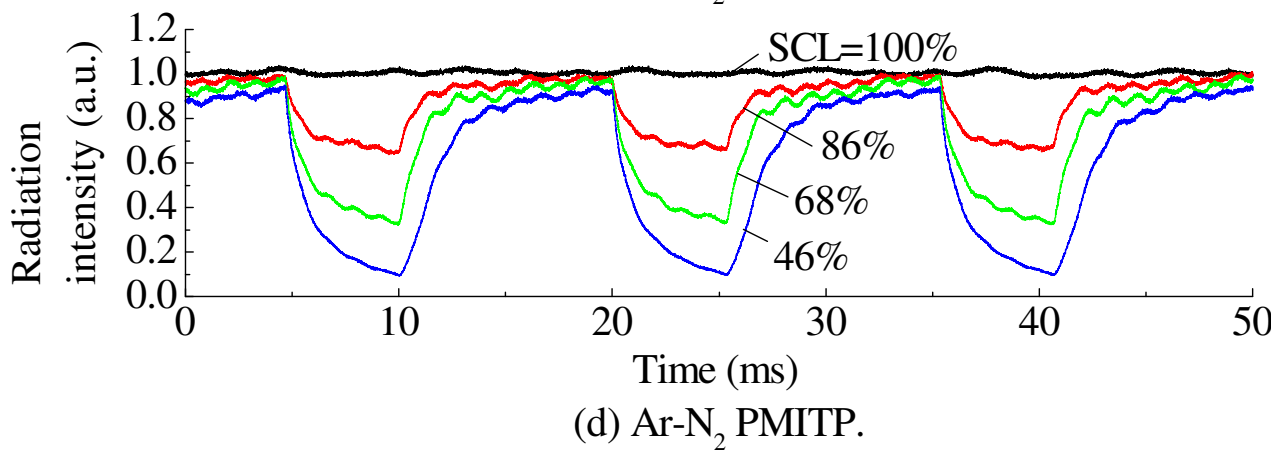

Figure 6. Time evolution in radiation intensities in an Ar spectral line at 751.465 $\mathrm{nm}$ measured at the observation position $\mathrm{P}$, which is located at the middle of the coil. (a) Pulsed signal. Waveforms for (b) Ar PMITP, (c) Ar- $\mathrm{H}_{2}$ PMITP and (d) Ar- $\mathrm{N}_{2}$ PMITP. 


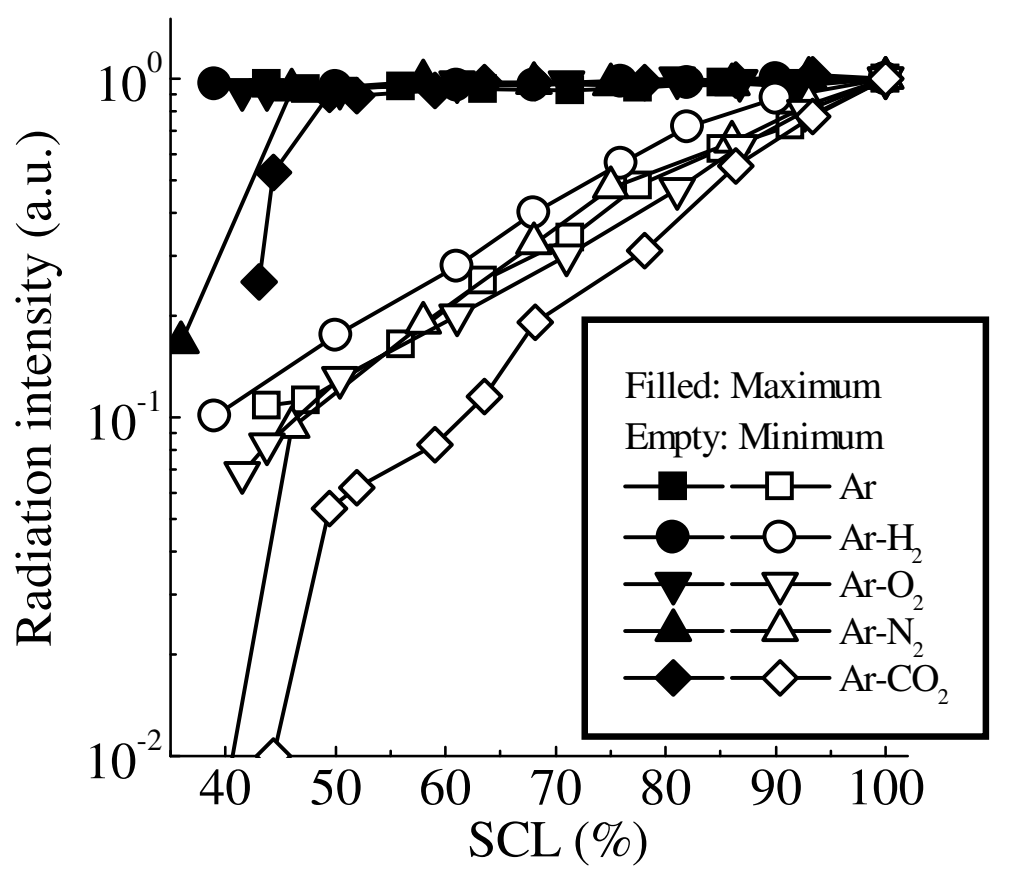

Figure 7. Effect of shimmer current level (SCL) on the maximum and minimum values of radiation intensity of Ar line at $751.465 \mathrm{~nm}$ in a modulation cycle measured at the observation position $\mathrm{P}$ for different gas-added PMITPs. 

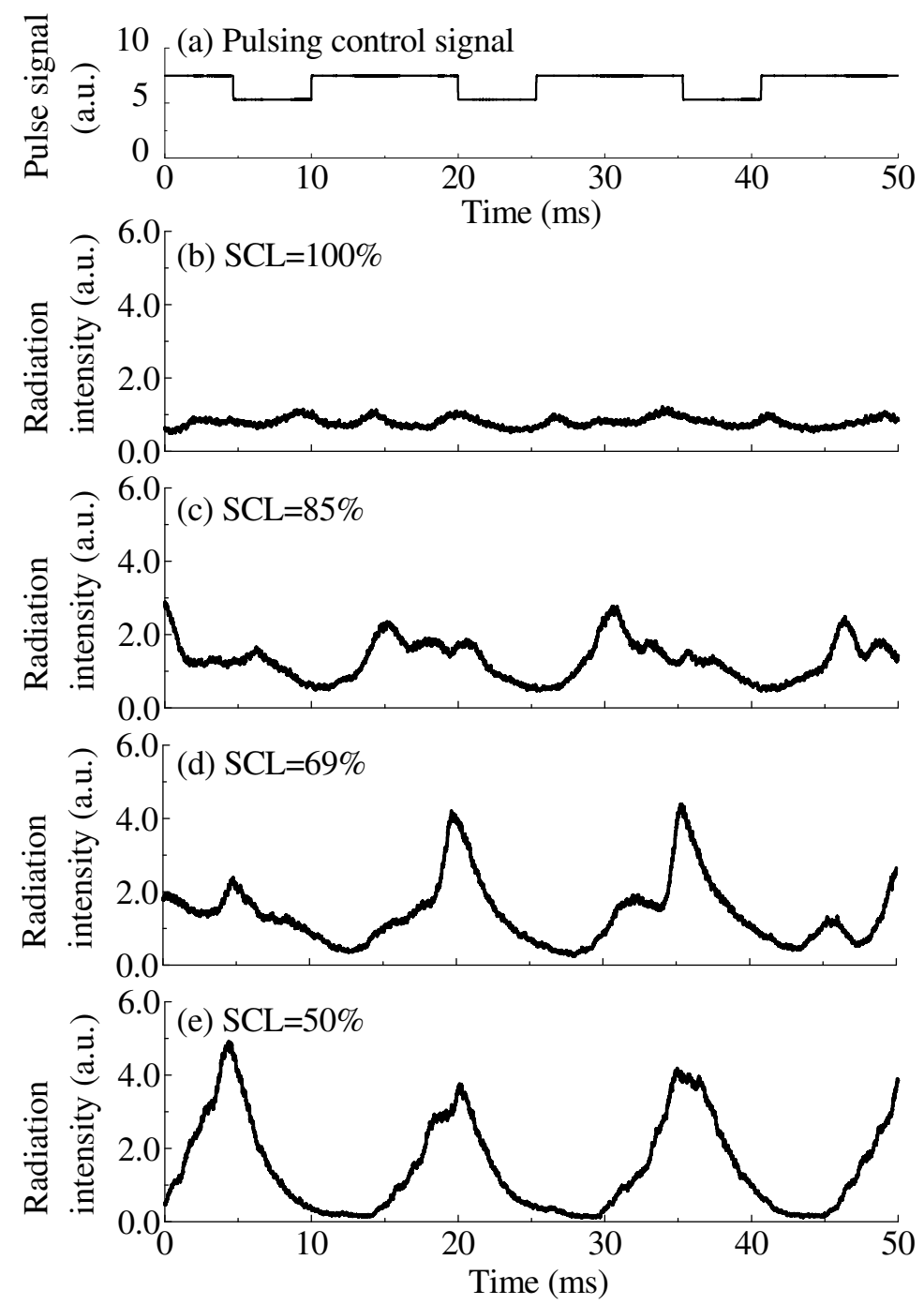

Figure 8. Time evolution in radiation intensities in an Ar spectral line at $751.465 \mathrm{~nm}$ measured at observation position $\mathrm{R}$ in Ar PMITP. (a) Pulsed signal. Intensities for (b) SCL $=100 \%$, (c) SCL $=85 \%$, (d) SCL $=69 \%$, (e) SCL $=50 \%$. 

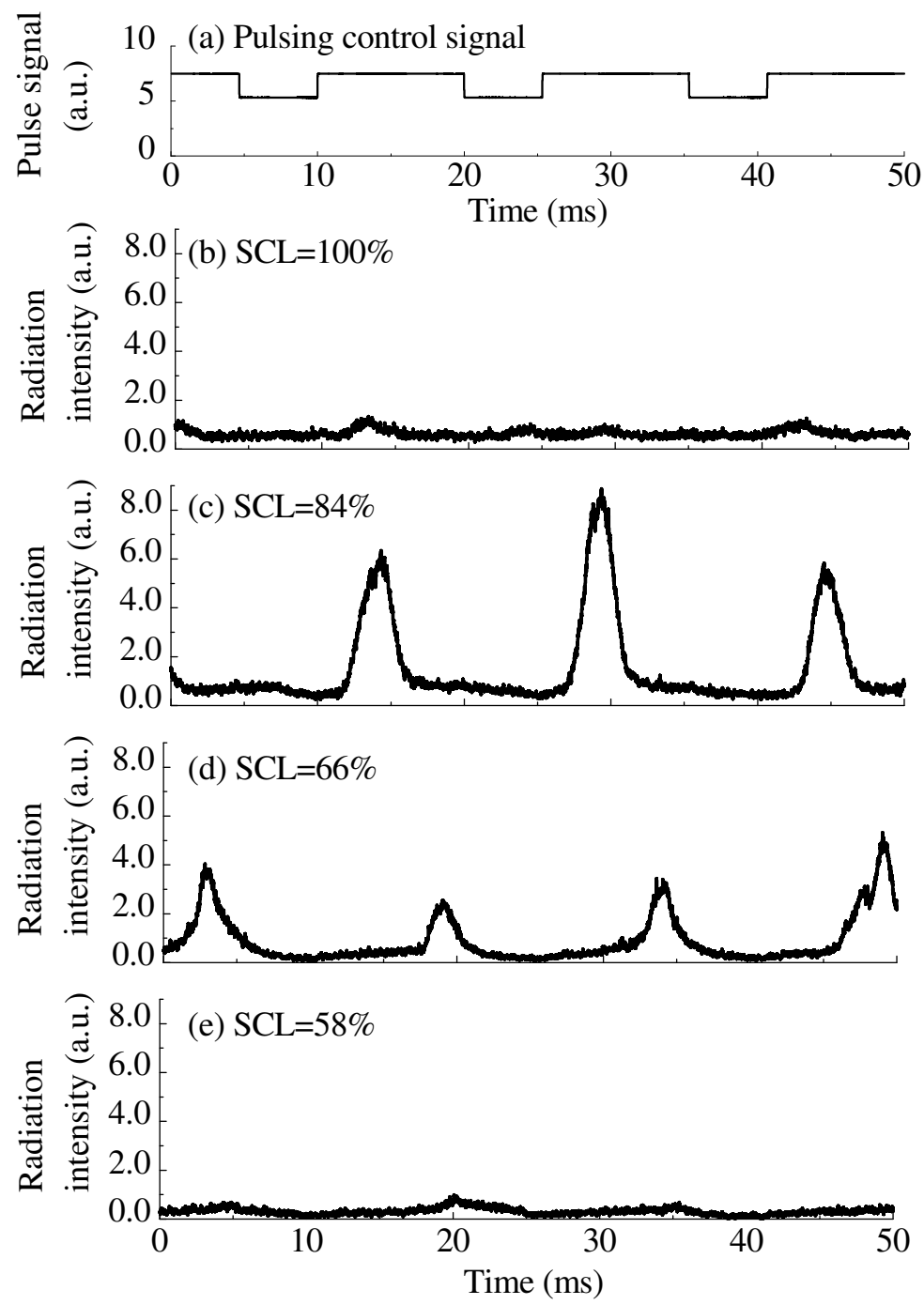

Figure 9. Time evolution in radiation intensities for an Ar spectral line at 751.465 $\mathrm{nm}$ measured at the observation position $\mathrm{R}$ in $\mathrm{Ar}-\mathrm{H}_{2}$ PMITP. (a) Pulsing signal. Waveforms for (b) SCL $=100 \%$, (c) SCL $=84 \%$, (d) SCL $=66 \%$, (e) SCL $=$ $49 \%$. 

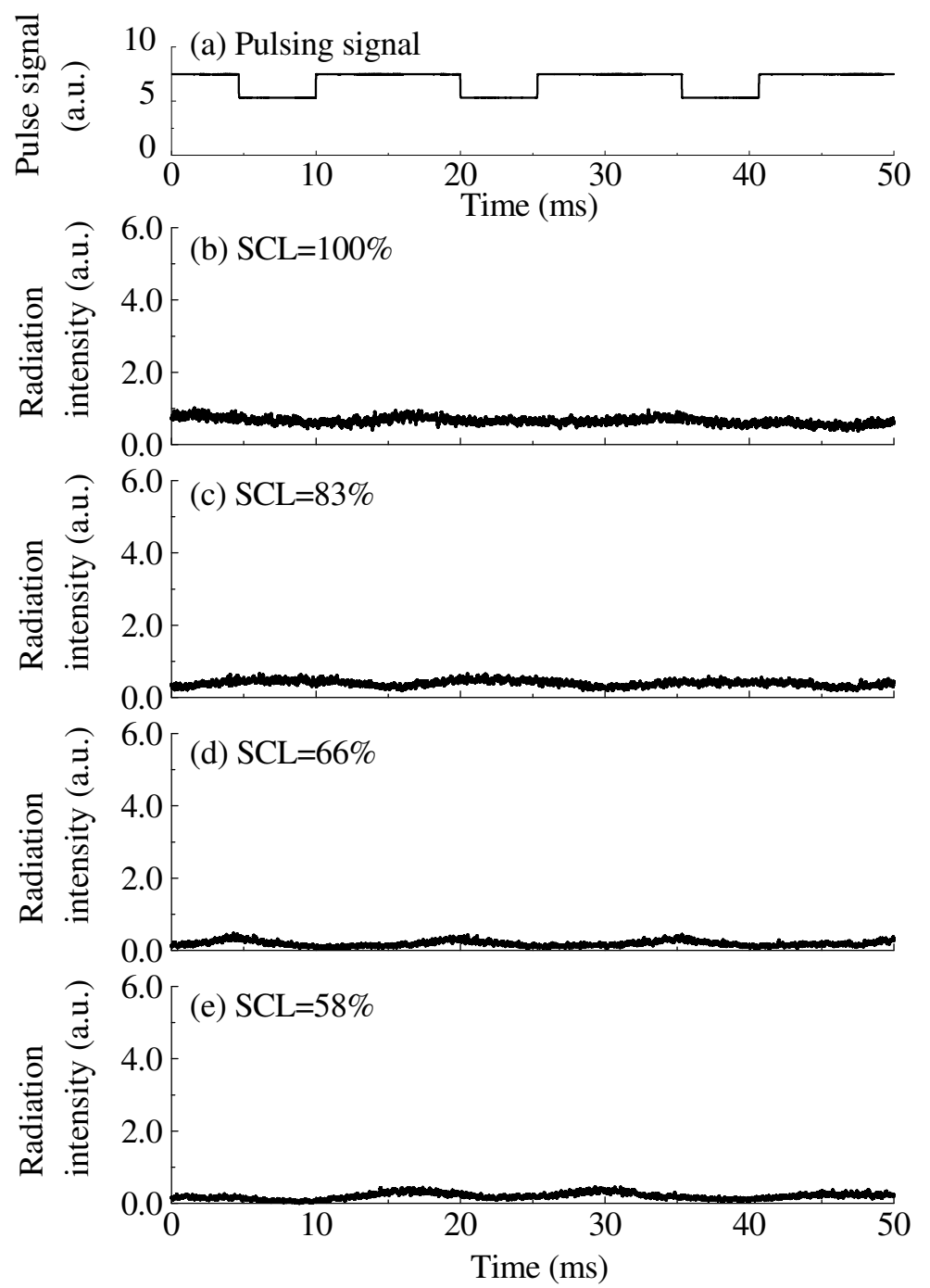

Figure 10. Time evolution in radiation intensities for an Ar spectral line at $751.465 \mathrm{~nm}$ measured at the observation position $\mathrm{R}$ in Ar- $\mathrm{N}_{2}$ PMITP. (a) Pulsed signal. Waveforms for (b) SCL $=100 \%$, (c) SCL $=83 \%$, (d) SCL $=66 \%$, (e) SCL $=58 \%$. 

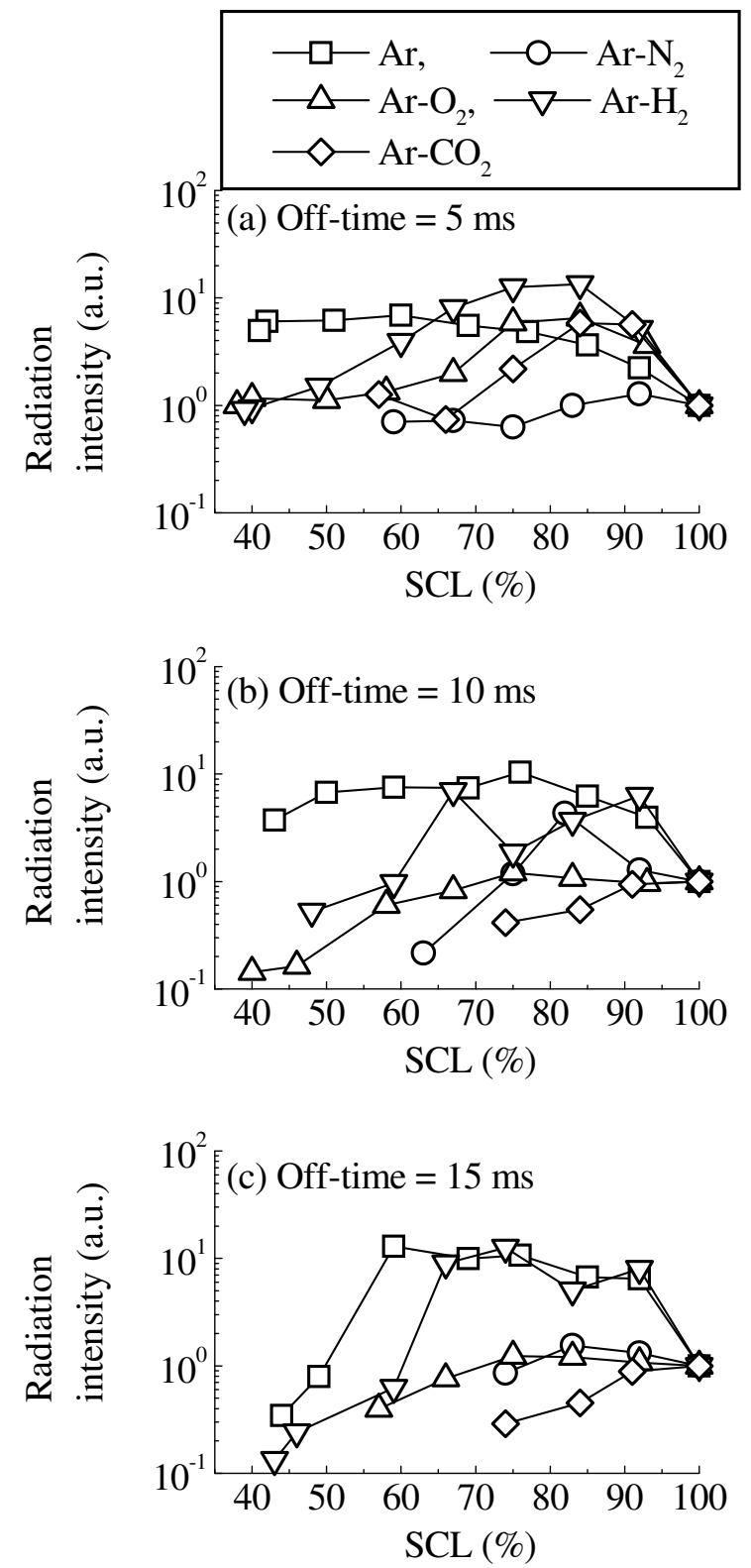

Figure 11. Normalized peak value of the radiation intensities of an Ar line at 751.465 $\mathrm{nm}$ measured at the observation position $\mathrm{R}$ as a function of shimmer current level (SCL). 


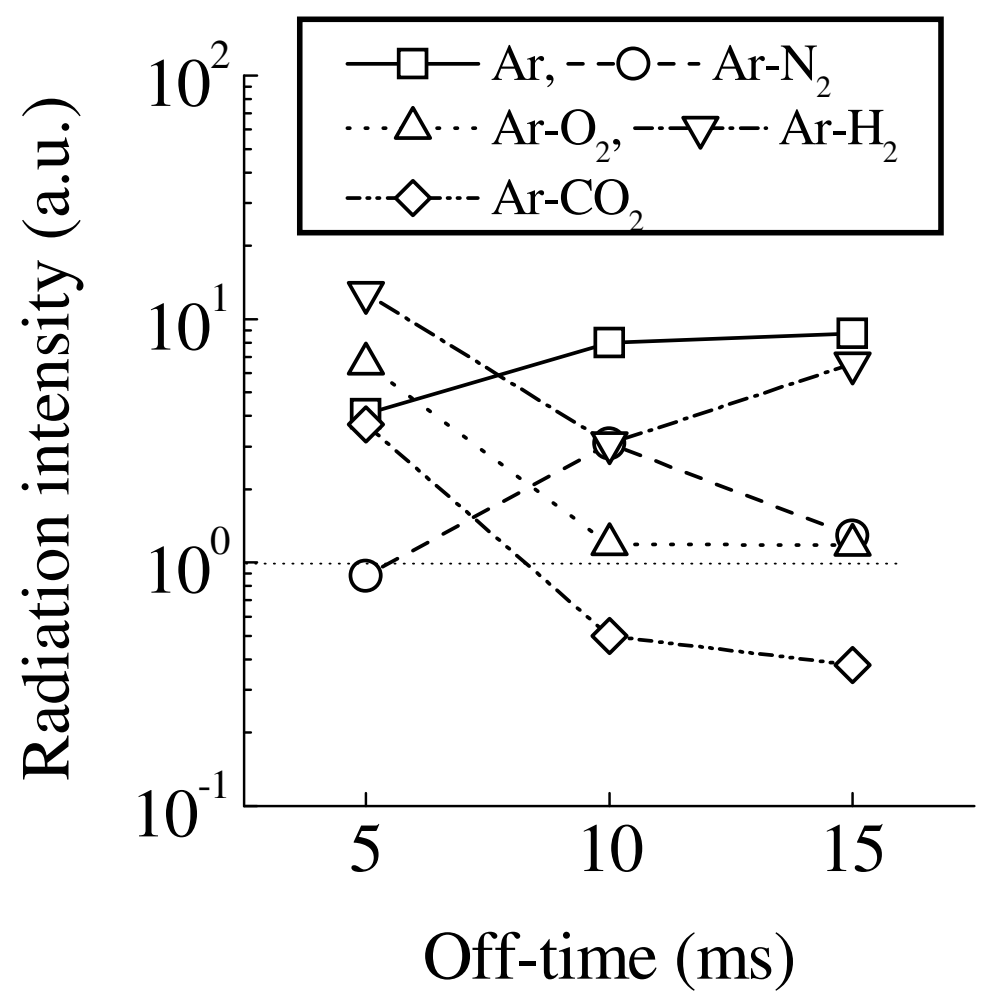

Figure 12. Dependence of the normalized radiation intensity peaks of an Ar line at $751.465 \mathrm{~nm}$ measured at the observation position R on the off-time for SCL $=80 \%$. 


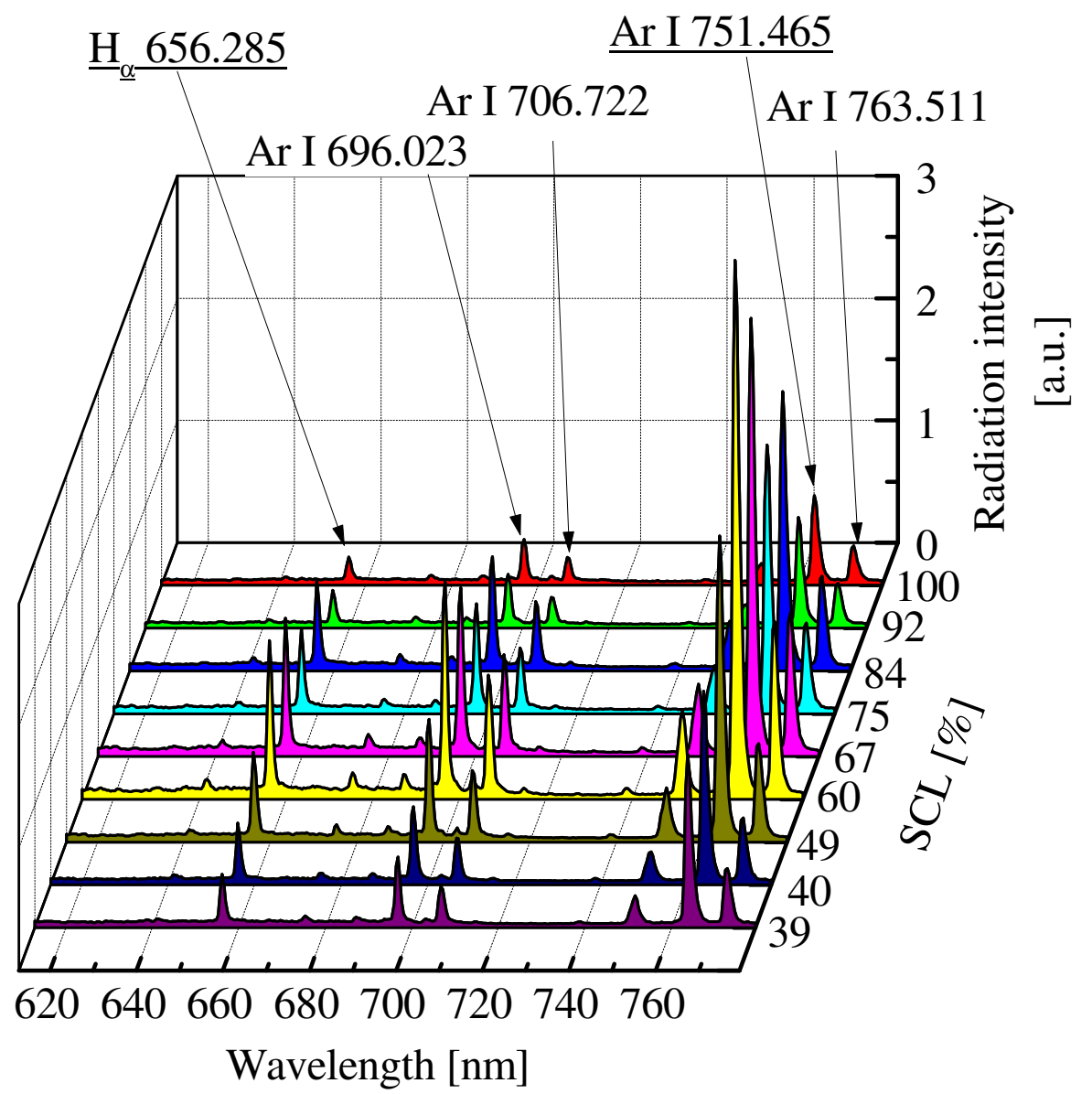

Figure 13. Time-accumulated radiation intensities in the wavelength range of 610-780 $\mathrm{nm}$ for $\mathrm{Ar}-\mathrm{H}_{2}$ PMITP with an off-time of $5 \mathrm{~ms}$. 


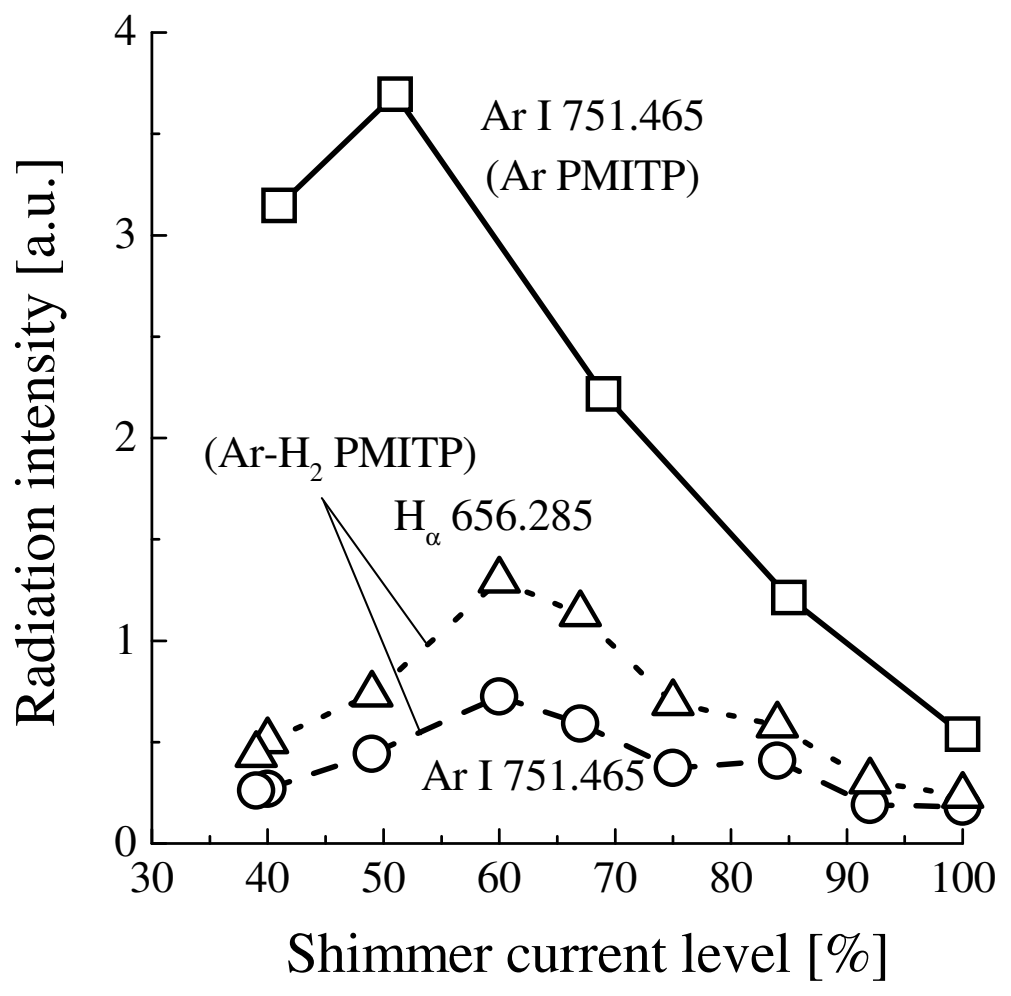

Figure 14. Effect of shimmer current level on time-accumulated radiation intensities of the $\mathrm{Ar}$ and $\mathrm{H}$ atomic lines for $\mathrm{Ar}$ and $\mathrm{Ar}-\mathrm{H}_{2}$ PMITPs. 